\title{
Graphenes Bonding Forces in Graphite
}

\author{
F. RozPŁOCH ${ }^{a}$, J. PATYK ${ }^{a}$ And J. StankOWski ${ }^{b}$ \\ ${ }^{a}$ Institute of Physics, Nicolaus Copernicus University \\ Grudziądzka 5/7, 87-100 Toruń, Poland \\ ${ }^{b}$ Institute of Molecular Physics, Polish Academy of Sciences \\ Smoluchowskiego 17, 60-179 Poznań, Poland
}

(Received September 4, 2007)

\begin{abstract}
Graphenes bonding forces in graphite are widely known as an example of the van der Waals forces. Well-known experimental facts relative to graphite negate this. A comparison of physical properties of graphite and molecular crystals and metals is shown. A model of dominant weak metallic bonding forces between graphenes is proposed. Brief theoretical background to the model is given.
\end{abstract}

PACS numbers: 73.20.--r, 81.05.Uw

\section{Introduction}

Graphite is a very well-known allotropic form of carbon. Carbon atoms in graphite planes are covalently bonded to three neighbours in plane with atoms placed in corners of hexagon. These planes are called graphenes. Lattice constants "a" and "b" in graphene are identical and equal to $0.2456 \mathrm{~nm}(2.456 \AA)$. A distance between neighbour atoms (length of bond) is $0.1418 \mathrm{~nm}(1.418 \AA)$.

Bonds between the graphenes are very weak. There are two types of graphite, which are called hexagonal and rhombohedral. The first type forms a sequence of $\mathrm{ABAB}$ graphene layers in which bonding energy is lower than in the second type (ABCABC sequence). This means that in the hexagonal graphite every second graphene layer is identical, that is all atoms have the same position in plane. In the rhombohedral graphite positions of atoms are identical in every third layer. The lattice constant in the direction perpendicular to graphene is $c=0.6694 \mathrm{~nm}$ $(6.694 \AA)$ for hexagonal type of graphite. A distance between neighbour graphenes is $c / 2=0.3347 \mathrm{~nm}(3.347 \AA)$. It is obvious that the hexagonal graphite is more common than the rhombohedral one.

Weak forces between graphenes suggest that they are the van der Waals forces. One can find such a conclusion in the Valence by Coulson [1]. As the van der Waals forces are forces between induced electric dipoles, there should 
be no electrical conductivity in the direction perpendicular to layers and very low thermal conductivity in this direction. Another conclusion is that molecular crystal graphite should have a low melting point temperature.

Bonding forces of graphene are very weak, comparable with these in molecular crystals. Such forces in molecular crystals are the van der Waals forces resulting from the induced polarity. Because of weak forces between graphenes it was assumed that they are the van der Waals forces.

And therefore, the prominent quantum chemist Coulson in his book from 1961 entitled Valence [1] wrote on page 391: "The distance between successive planes is $3.35 \AA$, a value so large that it can only arise from van der Waals forces". And further he wrote consequent but incorrect "... and in fact graphite does show a small electrical conductivity in its basal planes, but not across them". This is obviously wrong, because simple physical measurements deny this fact.

But also unfortunately in the Polish Physics Encyclopaedia [2] under the watchword "graphite structure" we can read "The atoms placed in the corners of regular hexagon with a side length of $0.142 \mathrm{~nm}$ are bonded by valence forces. The distance between the layers (graphenes) is $0.335 \mathrm{~nm}$. The layers are probably bonded by the weak van der Waals forces".

Even nowadays in scientific publications and handbooks the forces between graphenes are still described as the van der Waals forces.

\section{Experimental facts}

Let us remind what is typical of molecular (van der Waals) crystals:

1. Low melting point temperature (a few centigrade to about $300^{\circ} \mathrm{C}$ ).

2. Very small electrical conductivity (resistance of the order $10^{15} \Omega \mathrm{m}$ ).

3. Small thermal conductivity (order of $10^{-1} \mathrm{~W} \mathrm{~m}^{-1} \mathrm{~K}^{-1}$ ).

Graphite should show such features, if really bonding forces between graphenes would be of the van der Waals nature.

But for graphite we can observe:

1. Melting point about $4000 \mathrm{~K}$ under high pressure (under normal pressure over $3000 \mathrm{~K}$ it sublimates very fast).

2. Electrical conductivity along the graphene layers of the same order as for best conducting metals. Electrical conductivity in the direction perpendicular to the graphene layers about 300 times smaller than along them, but still much higher than that observed for semiconductors in room temperature.

3. Graphite shows relative good thermal conductivity and therefore it is used as a smearing material in the case when simultaneously good thermal conductivity is required.

On the basis of the above experimental facts, the model describing graphite as a molecular crystal, that is a crystal with the van der Waals forces as dominant forces between graphenes, has to be rejected. But similarly to the magnetic 
properties of solids, where a small admixture of diamagnetism is present in paramagnetics and ferromagnetics, a small admixture of the van der Waals forces may be present in the interaction between graphenes.

Schmidt [3] as the first considered a metallic model of graphite. Some other authors, for example Kuhn [4], involved a similar idea. All these papers aimed to find the relation between atomic and molecular orbitals in graphite. Cross interactions related to $\pi$-electrons were neglected in these papers. Our model of graphite involving metallic bonding forces (Fig. 1) was presented in a preliminary form in 1996 [5].

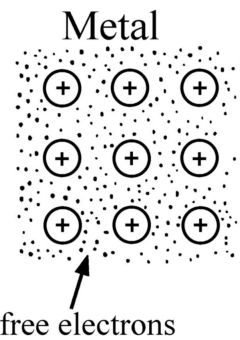

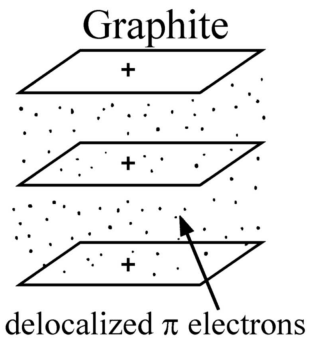

delocalized $\pi$ electrons

Fig. 1. Model of metallic graphite.

We assume in this model that there are weakly positive charged graphene layers in graphite playing a similar role as positive ions in crystal lattice of metals. Between graphenes delocalised $\pi$-electrons are moving. The quantity of the delocalised $\pi$-electrons is very small. We determined this on the basis of the analysis of $g$-factor in graphite and nanotubes [6] (see Table I, Fig. 2, and equations below):

$$
\begin{aligned}
& g_{m^{*}}=1+n\left(\frac{m_{\mathrm{e}}}{m_{\mathrm{e}}^{*}}\right)^{2}, \\
& m_{c}^{*}=\frac{m_{\mathrm{e}}}{\sqrt{\frac{g_{c}}{g_{a b}(1+1 / n)}-1 / n}},
\end{aligned}
$$

where $n$ is carrier concentration, $m^{*}$ — effective mass and indexed $g$ denotes values of $g$-factor in the configuration shown in Fig. 2 .

TABLE I

Values of $g$-factor and dynamical parameters in $s p^{2}$-bonded carbon materials. MWNT - multi-well carbon nanotubes.

\begin{tabular}{c|c|c|c}
\hline \hline & $g$-factor & $\begin{array}{c}\text { Carrier density } \\
{[\text { per atom }]}\end{array}$ & $\begin{array}{c}\text { Effective mass } \\
{\left[\mathrm{m}^{*} / \mathrm{m}_{\mathrm{e}}\right]}\end{array}$ \\
\hline Graphite & $\begin{array}{c}g_{c}=2.050 \\
g_{a b}=2.0026[7]\end{array}$ & $4.9 \times 10^{-5}[8]$ & $0.045[9]$ \\
\hline MWNT & $\begin{array}{c}g_{\perp}=2.0103 \\
g_{\|}=2.0137[10]\end{array}$ & $5.9 \times 10^{-5}[11]$ & $0.012[12]$ \\
\hline
\end{tabular}



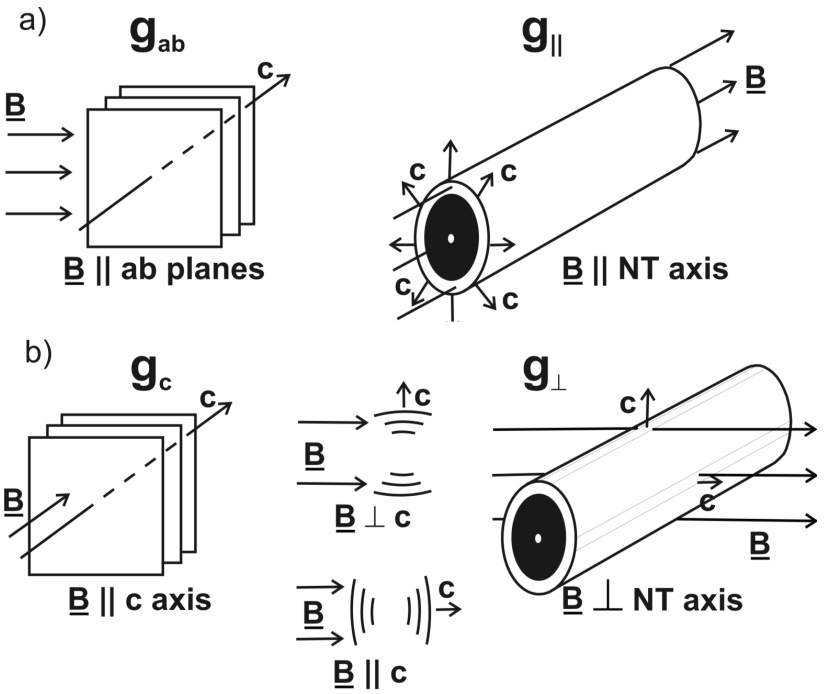

Fig. 2. Description of EPR measurement configuration for graphite and nanotubes.

The low concentration of quasi-free $\pi$-electrons in graphite (about $5 \times 10^{-5}$ electrons per atom) is a cause of weak metallic bond force between graphene layers. Because metallic forces in graphite are of the same order as for the van der Waals bonds, it was the origin of wrong conclusion that graphenes are bonded by the van der Waals forces.

The statement that the metallic model (model of very weak metallic forces) for graphene bonds is right, gives a good explanation of three observed physical properties of real graphite crystal (electrical conductivity in $c$ axis direction, melting point of graphite, thermal conductivity in $c$ axis direction).

Theoretical studies of Santos and Villagra [13] concerning the description of forces between graphene layers give a good support to this phenomenological model based on EPR investigations. They have used the Thomas-Fermi-Dirac (TFD) method including the full Kirznits correction. The correction describes the energy of definite electron configuration. The method is a kind of semi-empirical Hartree-Fock self confirmed field method.

This is the first method that gives a negative energy for the definite distance of two graphene layers (Fig. 3). The Kirznits correction used by authors is composed of two terms

$$
U_{k}=\frac{\hbar}{72 m} \int \frac{(\nabla \rho)^{2}}{\rho} \mathrm{d} V-\frac{\hbar}{12 m} \int \nabla^{2} \rho \mathrm{d} V
$$

where $\rho$ - volume density of electric charge, $V$ - volume.

The first term describes the case of inhomogeneities when charges are point-like, and the second one when charges are distributed on the plane, which is the case of graphite. 


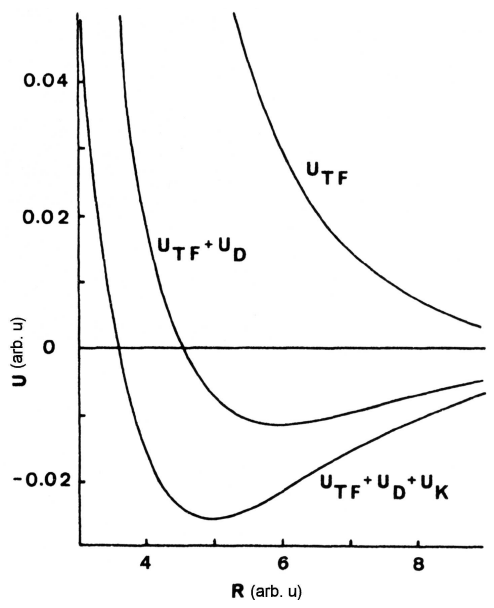

Fig. 3. Interlayer binding energy from Thomas-Fermi equations $\left\{U_{\mathrm{TF}}-\mathrm{TF}\right.$ energy, $U_{\mathrm{D}}$ - exchange (Dirac) energy, $U_{\mathrm{K}}$ - inhomogeneity (Kirzhnits) energy $\}$ [13].

The newest results of Novoselov et al. [14] for the Dirac fermions in graphene are giving a good support to our model of interlayer forces in graphite. We are convinced that in the future the model of metallic forces between graphene layers will be used as a dominant mechanism of bonding graphenes.

\section{Summary}

Graphite seems to be a very well-known material. Knowledge about its properties is not consistent with the theoretical description. A comparison of some physical properties of graphite to metals and molecular crystals is shown in Table II.

TABLE II

Graphite, molecular crystals and metals - comparison of some physical properties.

\begin{tabular}{c|c|c|c}
\hline \hline & Molecular crystal & Graphite & Metals \\
\hline $\begin{array}{c}\text { Electrical } \\
\text { resistance }\end{array}$ & $\approx 10^{23}$ & $\perp \boldsymbol{c}$ axis: $\approx 1.11$ & $1.6 \div 144$ \\
{$\left[10^{-8} \Omega \mathrm{m}\right]$} & $\begin{array}{c}\text { anthracene: } \\
10^{20}[15]\end{array}$ & $\boldsymbol{c}$ axis: $\approx \mathbf{3 0 0}[16]$ & $\begin{array}{c}\approx .3-\mathrm{Li}, \approx 1.6-\mathrm{Ag} \\
\approx 1.7-\mathrm{Cu}, \approx 21-\mathrm{Pb} \\
{[17,18]}\end{array}$ \\
\hline Melting & $273-573$ & $4000(10 \mathrm{MPa})[20]$ & $234 \div 3687$ \\
point & anthracene: & sublimation over & $453.7-\mathrm{Li}, 1235-\mathrm{Ag}$ \\
{$[\mathrm{K}]$} & $490[19]$ & $3000[21]$ & $1358-\mathrm{Cu}, 600.7-\mathrm{Pb}$ \\
& & & {$[17,18]$} \\
\hline $\begin{array}{c}\text { Thermal } \\
\text { conductivity }\end{array}$ & anthracene: & $\| \boldsymbol{c}$ axis: $\approx \mathbf{5 . 7}[18]$ & $401-\mathrm{Cu}, 35.3-\mathrm{Pb}$ \\
{$\left[\mathrm{W} \mathrm{m}^{-1} \mathrm{~K}^{-1}\right]$} & $0.5[22]$ & & {$[17,18]$}
\end{tabular}


This comparison leads to the conclusion that the graphene bonding forces are dominantly metallic not van der Waals. Theoretical calculations by Santos and Villagra [13] with the full Kirzhnits correction support this model. As a conclusion graphite is an anisotropic crystal with atoms covalently bonded in planes (graphenes) with the admixture of metallic bonding. Exceptionally good electrical transport is relative with extremely high mobility along planes. Graphenes are bonded by metallic forces with a very small concentration of electrons and relatively low mobility across graphenes. Metallic forces are a small admixture to the covalent forces in planes and they are dominant forces between planes (may be with a small admixture of the van der Waals forces).

\section{References}

[1] C.A. Coulson, Valence, 2nd ed., The University Press, Oxford 1965, p. 391.

[2] Physics Encyclopaedia, 1st ed., Vol. 1, PWN, Warszawa 1972, p. 710 (in Polish).

[3] O. Schmidt, Z. Phys. Chem. B 47, 1 (1940).

[4] H. Kuhn, Z. Elektrochem. 55, 220 (1951).

[5] F. Rozpłoch, Karbo 41, 111 (1996).

[6] F. Rozpłoch, J. Patyk, P. Szroeder, Mol. Phys. Rep. 37, 24 (2003).

[7] G. Wagoner, Phys. Rev. 118, 647 (1960).

[8] J.W. McClure, Phys. Rev. 112, 715 (1958).

[9] L.C. Tatar, S. Rabii, Phys. Rev. B 25, 4126 (1982).

[10] O. Chauwet, L. Forro, W. Bacsa, D. Ugarte, B. Doudin, W.A. de Heer, Phys. Rev. B 52, R6963 (1995).

[11] G. Baumgartner, M. Carrard, L. Zuppirioli, W. Bacsa, W.A. de Heer, L. Forro, Phys. Rev. B 55, 6704 (1997).

[12] I.L. Spain, K.J. Volin, H. Goldberg, I. Kalmin, J. Phys. Chem. Solids 44, 839 (1983).

[13] E. Santos, A. Villagra, Phys. Rev. B 6, 3134 (1972).

[14] K.S. Novoselov, A.K. Geim, S.V. Morozov, D. Jiang, M.I. Katsnelson, I.V. Grigorieva, S.V. Dubonos, Nature 438, 197 (2005).

[15] R.G. Kepler, Phys. Rev. 119, 1226 (1960).

[16] C.A. Klein, Rev. Mod. Phys. 34, 56 (1962).

[17] C. Kittel, Introduction to Solid State Physics, ed. 7, Wiley, New York 1996.

[18] CRC Handbook of Chemistry and Physics, 85th ed., CRC Press, Boca Roton 2004-2005, p. 12-45, 12-221.

[19] R.T. Morrison, R.N. Boyd, Organic Chemistry, 3rd ed., Allyn and Bacon, Inc., Boston 1978, p. 967.

[20] J. Basset, J. Phys. Rad. 10, 217 (1939).

[21] F.P. Bundy, J. Chem. Phys. 38, 618 (1963).

[22] R.G. Ross, P. Andersson, G. Backstrom, Mol. Phys. 39, 277 (1980). 